\title{
Rapid 3D whole-heart cine imaging using golden ratio stack of spirals
}

Javier Montalt-Tordera1, Grzegorz Kowalik1, Alexander Gotschy2,3, Jennifer Steeden1, Vivek Muthurangu 1

1 UCL Institute of Cardiovascular Science, UCL, London, UK

2 Great Ormond Street Hospital, London, UK

3 Institute for Biomedical Engineering, University and ETH Zurich, Zurich, Switzerland

All correspondence should be addressed to Javier Montalt-Tordera

(javier.tordera.17@ucl.ac.uk).

E-mail address for rest of authors:

Grzegorz Kowalik: grzegorz.kowalik.09@ucl.ac.uk

Alexander Gotschy: Alexander.Gotschy@usz.ch

Jennifer Steeden: jennifer.steeden@ucl.ac.uk

Vivek Muthurangu:v.muthurangu@ucl.ac.uk 


\section{INTRODUCTION}

Multi-slice breath-hold cine imaging using balanced steady state free precession (bSSFP) is the standard MRI method of assessing cardiac function and dynamic anatomy [1]. However, it is complex and time consuming. Acquisition of each slice must be segmented over several cardiac cycles, and breath-holding becomes necessary to avoid motion artefact. This can be problematic in children and patients with shortness of breath. In addition, multiple 2D slices must be acquired in several orientations in order to cover the anatomy of interest, further increasing total scan time.

Free-breathing whole-heart cine MRI is a desirable solution to these issues. This technique enables the acquisition of dynamic volumetric data with full anatomical coverage in a single, easy to plan scan, which can be reformatted in any plane during post processing. However, the acquisition of such high-dimensional data within a clinically feasible scan time demands extensive use of acceleration techniques and advanced reconstruction methods [2], such as parallel imaging [3] and compressed sensing [4,5].

Several 3D cine applications have been developed so far. Some are based on radial trajectories, such as spiral phyllotaxis koosh-ball [6-8] and stack of stars [9,10]. Cartesian approaches have also been used [11,12]. Spiral trajectories, however, have received little interest in this particular problem. The main advantage of spirals is the efficiency with which they cover $k$-space. Unlike radial trajectories, where sampling density varies quadratically with the radius, spirals have a relatively uniform (and adjustable) sampling density. As a result, they can sample $k$-space at the Nyquist rate (or at any given acceleration factor) using a significantly smaller number of readouts. Another source of efficiency is that readouts can be longer, which further decreases the number of excitations required, although this may not be as relevant for bSSFP sequences due to tight restrictions to TR [13]. The benefit of additional efficiency is that it can be leveraged to reduce acquisition time in data-intensive applications such as 3D cine imaging.

Spirals can be stacked in order to sample a volumetric $k$-space [14]. In particular, it has been recently shown that a golden ratio stack of spirals (GRASS) sampling strategy allows highly accelerated 3D plus time spoiled gradient echo imaging [15]. In addition to the efficiency of spirals, the use of golden ratio spacing in both the $k_{x}-k_{y}$ plane and along $k_{z}$ produces incoherent aliasing that is well suited to compressed sensing reconstruction. A further benefit is that the central $k_{z}$ line $\left(k_{x}, k_{y}=0\right)$ can be used for respiratory self-navigation, e.g. to gate the acquisition or to sort readouts into multiple respiratory phases [16]. However, GRASS utilises golden ratio 
spacing in $k$, between consecutive readouts. This is unsuitable for bSSFP sequences, which exhibit image artefacts when subject to large stepwise changes in eddy currents [17]. Therefore, alternative sampling strategies must be considered to adapt GRASS for use in a bSSFP pulse sequence, such as uniform and tiny golden ratio [18] sampling.

This work presents a rapid free-breathing whole-heart cine imaging framework based on a stack of spirals trajectory with a parallel imaging and compressed sensing reconstruction. The aims were to: (1) investigate the optimal ordering scheme for a stack of spirals cine sequence using an in silico model, (2) implement such a sequence and test it in vivo and (3) evaluate image quality and quantitative measurements against the gold standard examination, i.e. 2D multi-slice breath-hold imaging.

\section{MATERIALS AND METHODS}

This work is divided into two parts: (1) a simulation study comparing two different step sizes in $k z$, and (2) a patient study comparing the resulting technique to conventional 2D multi-slice breath-hold imaging.

\subsection{Sequence Design}

The sequence employed in this work is a 3D bSSFP stack of spirals acquired in a transverse orientation. A uniform density spiral trajectory was designed using the method described by Pipe et al. [19], assuming a field-of-view of $450 \mathrm{~mm}$, a spatial resolution of $2.0 \times 2.0 \mathrm{~mm}$ and 90 uniformly spaced spiral arms for complete filling of $k$-space, at each of the $104 k z$ partitions.

A golden ratio stack of spirals (GRASS) sampling strategy was used [15], which is based on a continuously running nested loop strategy. In the inner loop, consecutive spiral interleaves were advanced in the $k_{z}$ direction, while keeping their $k_{x}-k_{y}$ position the same. Each inner loop consisted of 104 steps and was followed by an outer loop rotation of the spiral arm by the golden angle $\left(\sim 222^{\circ}\right)[20]$ (Figure 1).

The following imaging parameters were used: TR/TE $=3.42 / 0.95 \mathrm{~ms}, \mathrm{FOV}=450 \times 450 \times 210$ $\mathrm{mm}_{3}$, matrix size $=224 \times 224 \times 104$, voxel size $=2.0 \times 2.0 \times 2.0 \mathrm{~mm}_{3}$, flip angle $=60^{\circ}$, bandwidth $=1,590 \mathrm{~Hz} /$ pixel. A total of 62,400 readouts were continuously acquired for a fixed scan time of $3 \mathrm{~min} 33 \mathrm{~s}$.

When using non-Cartesian trajectories such as spirals, some attention must be dedicated to ensuring trajectory fidelity. In modern scanners, the most important source of trajectory errors is the gradient delay relative to the ADC. This can be easily adjusted by appropriately shifting the ADC window and/or correcting the trajectory. The delay is typically different for each 
physical gradient axis [21]. However, if these differences are relatively small, as is the case for the scanner used in this work, using a single optimized delay affords most of the benefit while simplifying the implementation [22]. In the present work, the delay was set to the average of the delays in the $x$ and $y$ physical axes (ignoring $z$ as it was only used for phase encoding).

\subsection{Reconstruction}

The same reconstruction was used for both the simulation and patient studies. We expanded on the previously described GRASS framework [15], to develop XD-GRASS which sorts the data into cardiac and respiratory motion states, and reconstructs the undersampled multidimensional dataset using parallel imaging and compressed sensing, similar to [8]. This method has the advantage of exploiting sparsity in the respiratory dimension.

Data were retrospectively gated into 20 cardiac phases and 4 respiratory phases, totalling 80 volumes, with an acceleration factor of $\sim 12$. Cardiac gating was based on timestamps (provided from the vector cardiogram signal in the patient study, simulated timestamps in the simulation study). In order to obtain respiratory information, head-foot projections were retrospectively extracted from the $k$-space samples in the $k_{z}$ axis, both in the patient study and the simulation study (Figure 2). This has been previously described for stack of spirals data and validated against respiratory bellows [15]. A method based on principal component analysis (PCA) and Fourier analysis was then used to extract a respiratory signal from the projections [23]. Such a signal has been shown to be a reliable indicator of respiratory position. Finally, the respiratory signal was used to sort the $k$-space samples into respiratory bins from end-expiration to end-inspiration [16].

After data sorting, an iterative reconstruction combining parallel imaging and compressed sensing was used to obtain images from a subsampled $k$-space. Prior to the reconstruction, coil compression was performed in order to limit computational cost [24]. Then, coil sensitivity maps were obtained for the virtual coils using the ESPIRiT method [25].

After calibration, images $m$ were reconstructed from sorted $k$-space data $d$ by solving the following optimization problem [16]:

$$
m=\underset{m}{\operatorname{argmin}}\|F \cdot C \cdot m-d\|_{2}^{2}+\lambda_{c}\left\|D_{c} \cdot m\right\|_{1}+\lambda_{r}\left\|D_{r} \cdot m\right\|_{1}
$$

Where $F$ is the Fourier transform operator; $C$ is the coil sensitivity maps; $D_{c}$ and $D_{r}$ are the finite difference operators for the cardiac and respiratory dimensions, respectively; and $\lambda_{c}$ and $\lambda_{r}$ are the regularization parameters for the cardiac and respiratory dimensions, respectively. 
Regularization parameters were selected empirically based on visual inspection and set to 0.005 and 0.002 , respectively.

The reconstruction was implemented in MATLAB R2018a (The Mathworks, Inc, Natick, MA, USA). The Berkeley Advanced Reconstruction Toolbox (BART) was used for the ESPIRiT calibration and reconstruction [16]. Reconstructions were performed in a high-performance computing (HPC) cluster node, equipped with an 18-core Intel Xeon Gold 6140 processor and $432 \mathrm{~GB}$ of DDR4 memory.

\subsection{Simulation Study}

A simulation study was designed to investigate which step size in $k_{z}$ provides optimal filling in $k$-space, while meeting the requirements that: (1) it must be small enough so as not to cause eddy current related artefacts, and (2) it must allow the calculation of a respiratory navigator as described above. Two alternative step sizes were investigated: uniform spacing (US) and tiny golden spacing (TS), which used the 7th tiny golden ratio (0.116) [26] (Figure 1). It is worth noting that the problem with eddy currents does not apply to golden angle rotations in the outer loop, due to "pairing" compensation [17]. Therefore, the golden angle was used in the outer loop.

The design of the model was similar to the one described in [15]. Ellipsoids and cylinders were used to model the heart, liver, ascending/descending aorta, left/right pulmonary arteries and the thoracic wall (Figure 3). The heart and blood vessels were given contractile dynamics, while respiratory dynamics were modelled using a craniocaudal translation. Low frequency sinusoids were used to incorporate coil sensitivities into the model. Because all these elements have analytic representations in the Fourier domain, direct simulation of the $k$-space signal is possible [17]. This prevents the so-called inverse crime bias, which arises in rasterized simulations due to the use of identical models for both data synthesis and image reconstruction.

This model was used to generate multi-channel $k$-space data for both the US and the TS reordering schemes. Simulations were repeated four times at random heart rates (drawn from a uniform distribution between 60-100 bpm) and respiratory rates (12-18 bpm) to average out possible effects due to these factors. Data were "collected" and reconstructed as described in sections 2.1 and 2.2. In addition, reference images were obtained by simulating a fully sampled stack of spirals acquisition for every cardiorespiratory state. Root mean squared error (RMSE) and structural similarity (SSIM), were calculated against these reference images to assess image quality for both reordering strategies. 


\subsection{Patient Study}

\subsubsection{Data Acquisition}

Ten subjects were prospectively recruited for this study (age $21.2 \pm 10.1$ years). All were hospital patients for whom an MR scan had been clinically indicated. This study was approved by the local research ethics committee, and written consent was obtained from all subjects/guardians (Ref: 06/Q0508/124). A 12-channel receiver coil array (6-element body coil plus 6-element spine coil) was used and vector cardiogram time stamps were logged for retrospective cardiac gating. The proposed free-breathing whole-heart (FB-WH) cine method (see imaging protocol above) was compared with a conventional 2D multi-slice breath-hold stack in a short axis orientation (BH-SAX), with field of view $=\sim 340 \times 300 \mathrm{~mm} 2$, voxel size $=$ $\sim 1.5 \times 1.5 \mathrm{~mm}$, slice thickness $=8.0 \mathrm{~mm}$, flip angle $=70^{\circ}, 10-12$ slices, temporal resolution $=\sim 40 \mathrm{~ms}$. All imaging was performed on a 1.5 T MR scanner (Avanto, Siemens Healthineers AG, Erlangen, Germany).

\subsubsection{Data Processing}

All post processing was performed using the OsiriX open source DICOM viewing platform (OsiriX v9.0, OsiriX Foundation, Geneva, Switzerland) [27]. Prior to analysis, FB-WH volumes were reformatted into a stack of short axis cines with a slice thickness of $8 \mathrm{~mm}$ using the multiplanar functionality in OsiriX, in order to obtain a dataset comparable to the BH-SAX.

Image Quality Assessment. Image quality was assessed on the mid-ventricular slice of the $\mathrm{BH}-\mathrm{SAX}$ data and the short axis reformatted FB-WH data. Quantitative image quality was evaluated using two objective scores: edge sharpness (ES) and contrast-to-noise ratio (CNR). The ES was calculated by drawing a line across the endocardial border, fitting a 10th order polynomial to the normalized pixel intensities (to filter out noise), and taking the maximum gradient of the fitted line as the measurement, as previously described [28]. The CNR was calculated as the ratio of the average signal difference between blood pool and myocardium to the average standard deviation in both regions [12]. The regions of interest for blood and myocardium were manually positioned in the centre of the left-ventricular cavity and the ventricular septum, respectively. In addition, subjective image quality was rated by a clinical observer on a 5 -point Likert scale $(0=$ non-diagnostic, $1=$ poor, $2=$ moderate, $3=$ good, $4=$ excellent) in three categories: sharpness of the endocardial border, temporal fidelity of wall motion and residual artefacts. 
Ventricular Volume Quantification. Quantification of left ventricular (LV) and right ventricular (RV) volumes was performed in a similar manner for each technique using in-house plugins for OsiriX by a single observer (AG, 7 years of experience in CMR). Firstly, the end-diastolic and end-systolic phases were identified for each ventricle through visual inspection of the midventricular cine. The endocardial borders of all slices at end systole and diastole were then traced manually. This allowed calculation of end-diastolic volume (EDV) and end-systolic volume (ESV). Stroke volume (SV) was obtained by subtracting ESV from EDV and ejection fraction $(E F)=S V / E D V \times 100$. The observer was presented with each anonymized volume in a random order, blinded to diagnosis, patient number and type of sequence.

\subsection{Statistical Analysis}

All statistical analysis was performed using $R$ software ( $R$ Foundation for Statistical Computing, Vienna, Austria) and results were considered statistically significant if $p<0.05$. In the simulation study, $t$-tests were used to assess the hypothesis that RMSE and SSIM values from the US and TS images have equal means. In the patient study, edge sharpness and CNR scores for the FB-WH and BH-SAX methods were compared using t-tests, while qualitative scores were compared using Wilcoxon signed-rank tests. Bland-Altman analysis was used to compare ventricular volume measurements between FB-WH and BH-SAX.

\section{RESULTS}

\subsection{Simulation Study}

Figure 3 shows representative axial and coronal views of the reconstructed phantom images for the US and TS sampling strategies. Visual inspection reveals better image quality in the case of US sampling, with less artefact, sharper edges and less temporal blurring. This is particularly obvious in the coronal view. The RMSE measure seems to agree with this observation (US: $0.0472 \pm 0.0005$ vs TS: $0.0482 \pm 0.0007, p=0.0852$ ), as well as SSIM (US: $0.8937 \pm 0.0217$ vs TS: $0.8608 \pm 0.0193, p=0.0954$ ). Since the use of TS does not seem to offer any benefits, US was used for the patient study.

\subsection{Patient Study}

FB-WH and BH-SAX data were acquired successfully in all patients. Acquisition time for FBWH was $3 \min 33 \mathrm{~s}$, compared to $4 \min 48 \mathrm{~s} \pm 51 \mathrm{~s}$ for $\mathrm{BH}-\mathrm{SAX}(\mathrm{p}<0.05)$. The average heart rate during the scans was $71 \pm 13 \mathrm{bpm}$. Reconstruction time for FB-WH images was 105.6 $\min \pm 3.3 \mathrm{~min}$ per patient. Representative end-systole and end-diastole images are shown in Figure 4, in a short axis orientation (multiplanar reformatted) for comparison with BH-SAX. Coronal views are shown in Figure 5 to illustrate the depiction of respiratory motion. 
Subjective image quality scores were lower for FB-WH data compared to BH-SAX as shown in Table 1, but on average were still in the clinically useful range. Quantitative edge sharpness of the FB-WH images was significantly lower than the BH-SAX $(0.13 \pm 0.03$ vs $0.64 \pm 0.18$ $\mathrm{mm}-1, p<0.05)$. CNR was also lower $(13.8 \pm 4.7$ vs $16.4 \pm 5.4)$ but this did not reach statistical significance $(p=0.29)$.

Table 2 compares the volumes obtained from the FB-WH and $\mathrm{BH}-\mathrm{SAX}$ acquisitions. FB-WH slightly underestimated end diastolic volumes for both ventricles (left: $-4.0 \mathrm{~mL},-2.7 \%$; right: $-8.2 \mathrm{~mL},-4.6 \%$ ) and overestimated end systolic volumes (left: $+5.3 \mathrm{~mL},+9.3 \%$; right: +2.6 $\mathrm{mL},+3.7 \%)$. The remaining measures are derived from these and thus reflect the same biases. The corresponding Bland-Altman plots are shown in Figure 6.

\section{DISCUSSION}

This work presents a stack of spirals free-breathing whole-heart cine sequence, which leverages the golden ratio to impart incoherence to the aliasing artefacts as necessary for compressed sensing reconstruction. The main findings of this study were: (1) stack of spirals free-breathing whole-heart cine imaging was feasible in patients, (2) uniform spacing in $k_{z}$ was preferable to tiny golden step spacing in in silico models, (3) image quality was lower than that of breath-hold short axis stacks, but was still in the clinically useful range, and (4) there was reasonable agreement between ventricular volumes measured using FB-WH and BH-SAX images. Thus, this sequence offers a rapid, easy to plan free breathing alternative to multislice breath-hold imaging in patients with heart disease.

The sequence used in this study is based on a previously described golden ratio stack of spirals (GRASS) sequence used for contrast enhanced angiography. The use of golden ratio steps in $k_{z}$ and golden angle rotations in $k_{x}-k_{y}$ promotes both incoherent aliasing and optimized $k$-space filling after retrospective cardio-respiratory binning. However, this sampling pattern is poorly suited to bSSFP imaging due to the large jumps in $k$, which would result in significant eddy current artefacts. Two plausible alternatives are uniform spacing and tiny golden ratio spacing, both of which have smaller step sizes, but still allow formation of a respiratory selfnavigation signal. In our in silico model, no evidence could be found that the tiny golden ratio outperforms uniform spacing. This is slightly surprising because golden ratio spacing has been shown to outperform uniform spacing in $k$, when using spoiled gradient echo imaging [15]. The new observation may be explained by the fact that there are many more readouts (and rotations) in a bSSFP acquisition, since each readout must be kept shorter to minimize dark band artefacts. This may increase the incoherence provided by the golden angle rotations and 
reduce the dependence on non-uniform sampling in $k z$. Due to the better image quality in the in silico models, uniform spacing was used for the patient arm of the study.

In the patient study, the proposed scheme was on average $26 \%$ faster to acquire than the short axis stack. However, the short axis stack did have higher in-plane resolution, making direct comparison difficult. A more important benefit of the FB-WH technique is that it provides whole-heart coverage with isotropic resolution, enabling multiplanar reformatting (MPR) in arbitrary orientations during postprocessing. This reduces the need for multiple scans in different orientations (short axis, long axis, 4-chamber, outflow tracts, etc.), which are a timeconsuming element of conventional cardiac MR protocols. An additional advantage is that FBWH does not require breath-holding, which improves patient comfort.

The image quality of FB-WH data was lower than for BH-SAX images in terms of CNR, edge sharpness and subjective scores. There are several possible causes of lower image quality in FB-WH data. Firstly, in-plane resolution was lower in FB-WH compared to BH-SAX images, which reduces edge sharpness, as well as subjective image quality. Acquiring images with higher resolution is possible but would incur a significant cost in terms of scan and reconstruction times. Secondly, FB-WH acquisitions were highly accelerated, which makes the recovery of high-quality images challenging. Image quality could be improved by simply reducing the amount of acceleration, but this would also increase acquisition time. A better approach might be to try to improve the reconstruction itself. This could be done by using alternative sparsifying transforms [29], or incorporating low-rank constraints [30-32]. Thirdly, there was no fat suppression in our implementation of the FB-WH sequence. This is important as subcutaneous fat is a significant contributor to the aliasing artefact and increases the difficulty of reconstruction. Techniques such as fat saturation [33], alternating TR [34] or phase detection [35] can be used to attenuate signal from fat, though some would also incur an acquisition time penalty. Fourthly, spiral imaging is highly sensitive to off-resonance artefact, which manifests as blurring. It should be noted that this type of blurring increases with readout time as off-resonant spins accumulate phase. Thus, its influence is not expected to be very significant in this work, which uses a short readout time. Nevertheless, existing off-resonance correction methods [36-38] may offer some improvement. Finally, this work used a noncentred $T E$, i.e. $T E \neq T R / 2$. This was necessary in order to maintain scanning efficiency but is suboptimal in bSSFP sequences. Using a modified trajectory, such as spiral-in/out, would enable the use of a centred TE while maintaining efficiency [39]. Aligning TE to the spin refocusing at TR/2 should improve the signal-to-noise ratio and reduce blurring due to offresonant spins [13]. 
Although the FB-WH images had lower image quality than the BH-SAX data, there was still good agreement in the measurement of ventricular volumes. However, with the FB-WH technique there was a small but statistically significant underestimation of end diastolic volume and overestimation of end systolic volume, for both ventricles. This is probably due to temporal blurring and there are two possible causes. Firstly, as part of the compressed sensing reconstruction, significant amounts of temporal regularization are required to minimize the undersampling artefact. Secondly, although true temporal resolution is similar for both techniques ( $40 \mathrm{~ms}$ ), BH-SAX $k$-space data were interpolated during reconstruction to a finer temporal grid (40 reconstructed images, or about $20 \mathrm{~ms}$ per frame). This is not possible with the FB-WH technique because $k$-space coordinates are continuously rotated using the golden angle.

The results reported here are consistent with other free-breathing isotropic 3D cine techniques. One study [8], using a radial koosh-ball trajectory, achieves better spatial resolution and image quality, but scan time is about four times longer than in this work. Other Cartesian techniques that offer the same spatial resolution $[11,12]$ report more similar results in terms of image quality, agreement of ventricular volumes and scan time, but are still slower than the technique reported in this work. For the interested reader, a detailed discussion is given in Supplementary Information S1.

Finally, an important limitation of this work is the long reconstruction time and the necessary computing power. In order to enable clinical implementation, much optimization would be needed in terms of speed and memory usage, which in this work prevents the use of GPU computing. Incorporating new reconstruction technologies, such as those based on deep learning, could potentially improve the prospects in this regard.

\section{CONCLUSION}

In conclusion, this work presents a whole-heart imaging framework for comprehensive cardiac assessment in free-breathing conditions, with trivial planning and short acquisition time $(<4$ $\min )$. While the proposed method could not provide the same image quality as a conventional multi-slice breath-hold acquisition, several factors have been identified that could mitigate this. Long reconstruction time and high computational requirements are important challenges to be addressed. Addressing these limitations, the approach may represent a promising way to simplify and accelerate the cardiac MRI workflow. 


\section{ACKNOWLEDGMENTS}

We would like to express our gratitude to our clinical and research CMR radiographers in Great Ormond Street Hospital, London. This work was supported by Heart Research UK and the National Institute for Health Research (NIHR) Biomedical Research Centre at Great Ormond Street Hospital for Children National Health Service (NHS) Foundation Trust and University College London.

The authors would also like to acknowledge the use of the UCL Myriad High Performance Computing Facility (Myriad@UCL), and associated support services, in the completion of this work. 


\section{REFERENCES}

[1] Judd RM. Cardiac cine imaging. Basic Princ. Cardiovasc. MRI Phys. Imaging Tech., Springer International Publishing; 2015, p. 145-59. https://doi.org/10.1007/978-3-31922141-0_10.

[2] Jackson JI, Meyer CH, Nishimura DG, Macovski A. Selection of a convolution function for Fourier inversion using gridding (computerised tomography application). IEEE Trans Med Imaging 1991;10:473-8. https://doi.org/10.1109/42.97598.

[3] Deshmane A, Gulani V, Griswold MA, Seiberlich N. Parallel MR imaging. J Magn Reson Imaging 2012;36:55-72. https://doi.org/10.1002/jmri.23639.

[4] Lustig M, Donoho D, Pauly JM. Sparse MRI: The application of compressed sensing for rapid MR imaging. Magn Reson Med 2007;58:1182-95. https://doi.org/10.1002/mrm.21391.

[5] Jaspan ON, Fleysher R, Lipton ML. Compressed sensing MRI: A review of the clinical literature. Br J Radiol 2015;88. https://doi.org/10.1259/bjr.20150487.

[6] Piccini D, Littmann A, Nielles-Vallespin S, Zenge MO. Spiral phyllotaxis: The natural way to construct a 3D radial trajectory in MRI. Magn Reson Med 2011;66:1049-56. https://doi.org/10.1002/mrm.22898.

[7] Coppo S, Piccini D, Bonanno G, Chaptinel J, Vincenti G, Feliciano H, et al. Free-running 4D whole-heart self-navigated golden angle MRI: Initial results. Magn Reson Med 2015;74:1306-16. https://doi.org/10.1002/mrm.25523.

[8] Feng L, Coppo S, Piccini D, Yerly J, Lim RP, Masci PG, et al. 5D whole-heart sparse MRI. Magn Reson Med 2018;79:826-38. https://doi.org/10.1002/mrm.26745.

[9] Liu J, Spincemaille P, Codella NCF, Nguyen TD, Prince MR, Wang Y. Respiratory and cardiac self-gated free-breathing cardiac CINE imaging with multiecho 3D hybrid radial SSFP acquisition. Magn Reson Med 2010;63:1230-7. https://doi.org/10.1002/mrm.22306.

[10] Zhang X, Xie G, Lu N, Zhu Y, Wei Z, Su S, et al. 3D self-gated cardiac cine imaging at 3 Tesla using stack-of-stars bSSFP with tiny golden angles and compressed sensing. Magn Reson Med 2019;81:3234-44. https://doi.org/10.1002/mrm.27612.

[11] Usman M, Ruijsink B, Nazir MS, Cruz G, Prieto C. Free breathing whole-heart 3D CINE 
MRI with self-gated Cartesian trajectory. Magn Reson Imaging 2017;38:129-37. https://doi.org/10.1016/J.MRI.2016.12.021.

[12] Moghari MH, Barthur A, Amaral ME, Geva T, Powell AJ. Free-breathing whole-heart $3 \mathrm{D}$ cine magnetic resonance imaging with prospective respiratory motion compensation. Magn Reson Med 2018;80:181-9. https://doi.org/10.1002/mrm.27021.

[13] Scheffler K, Lehnhardt S. Principles and applications of balanced SSFP techniques. Eur Radiol 2003;13:2409-18. https://doi.org/10.1007/s00330-003-1957-x.

[14] Irarrazabal P, Nishimura DG. Fast three dimensional magnetic resonance imaging. Magn Reson Med 1995;33:656-62. https://doi.org/10.1002/mrm.1910330510.

[15] Kowalik GT, Steeden JA, Atkinson D, Montalt-Tordera J, Mortensen KH, Muthurangu V. Golden ratio stack of spirals for flexible angiographic imaging: Proof of concept in congenital heart disease. Magn Reson Med 2019;81. https://doi.org/10.1002/mrm.27353.

[16] Feng L, Axel L, Chandarana H, Block KT, Sodickson DK, Otazo R. XD-GRASP: Golden-angle radial $\mathrm{MRI}$ with reconstruction of extra motion-state dimensions using compressed sensing. Magn Reson Med 2016;75:775-88. https://doi.org/10.1002/mrm.25665.

[17] Bieri O, Markl M, Scheffler K. Analysis and compensation of eddy currents in balanced SSFP. Magn Reson Med 2005;54:129-37. https://doi.org/10.1002/mrm.20527.

[18] Wundrak S, Paul J, Ulrici J, Hell E, Rasche V. A small surrogate for the golden angle in time-resolved radial MRI based on generalized Fibonacci sequences. IEEE Trans Med Imaging 2015;34:1262-9. https://doi.org/10.1109/TMI.2014.2382572.

[19] Pipe JG, Zwart NR. Spiral trajectory design: A flexible numerical algorithm and base analytical equations. Magn Reson Med 2014;71:278-85. https://doi.org/10.1002/mrm.24675.

[20] Winkelmann S, Schaeffter T, Koehler T, Eggers H, Doessel O. An optimal radial profile order based on the golden ratio for time-resolved MRI. IEEE Trans Med Imaging 2007;26:68-76. https://doi.org/10.1109/TMI.2006.885337.

[21] Aldefeld B, Börnert P. Effects of gradient anisotropy in MRI. Magn Reson Med 1998;39:606-14. https://doi.org/10.1002/mrm.1910390414. 
[22] Bernstein MA, King KF, Zhou XJ. Handbook of MRI pulse sequences. Academic Press; 2004.

[23] Pang J, Sharif B, Fan Z, Bi X, Arsanjani R, Berman DS, et al. ECG and navigator-free four-dimensional whole-heart coronary MRA for simultaneous visualization of cardiac anatomy and function. Magn Reson Med 2014;72:1208-17. https://doi.org/doi:10.1002/mrm.25450.

[24] Huang F, Vijayakumar S, Li Y, Hertel S, Duensing GR. A software channel compression technique for faster reconstruction with many channels. Magn Reson Imaging 2008;26:133-41. https://doi.org/10.1016/J.MRI.2007.04.010.

[25] Uecker M, Lai P, Murphy MJ, Virtue P, Elad M, Pauly JM, et al. ESPIRiT—an eigenvalue approach to autocalibrating parallel MRI: Where SENSE meets GRAPPA. Magn Reson Med 2014;71:990-1001. https://doi.org/doi:10.1002/mrm.24751.

[26] Wundrak S, Paul J, Ulrici J, Hell E, Geibel M-A, Bernhardt P, et al. Golden ratio sparse MRI using tiny golden angles. Magn Reson Med 2016;75:2372-8. https://doi.org/10.1002/mrm.25831.

[27] Rosset A, Spadola L, Ratib O. OsiriX: An open-source software for navigating in multidimensional DICOM images. J Digit Imaging 2004;17:205-16. https://doi.org/10.1007/s10278-004-1014-6.

[28] Steeden JA, Atkinson D, Hansen MS, Taylor AM, Muthurangu V. Rapid flow assessment of congenital heart disease with high-spatiotemporal-resolution gated spiral phase-contrast MR imaging. Radiology 2011;260:79-87. https://doi.org/10.1148/radiol.11101844.

[29] Feng L, Benkert T, Block KT, Sodickson DK, Otazo R, Chandarana H. Compressed sensing for body MRI. J Magn Reson Imaging 2017;45:966-87. https://doi.org/10.1002/jmri.25547.

[30] Lingala SG, Hu Y, Dibella E, Jacob M. Accelerated dynamic MRI exploiting sparsity and low-rank structure: k-t SLR. IEEE Trans Med Imaging 2011;30:1042-54. https://doi.org/10.1109/TMI.2010.2100850.

[31] Otazo R, Candès E, Sodickson DK. Low-rank plus sparse matrix decomposition for accelerated dynamic MRI with separation of background and dynamic components. Magn Reson Med 2015;73:1125-36. https://doi.org/doi:10.1002/mrm.25240. 
[32] He J, Liu Q, Christodoulou AG, Ma C, Lam F, Liang ZP. Accelerated high-dimensional MR imaging with sparse sampling using low-rank tensors. IEEE Trans Med Imaging 2016;35:2119-29. https://doi.org/10.1109/TMI.2016.2550204.

[33] Scheffler K, Heid O, Hennig J. Magnetization preparation during the steady state: Fatsaturated 3D TrueFISP. Magn Reson Med 2001;45:1075-80. https://doi.org/10.1002/mrm.1142.

[34] Leupold J, Hennig J, Scheffler K. Alternating repetition time balanced steady state free precession. Magn Reson Med 2006;55:557-65. https://doi.org/10.1002/mrm.20790.

[35] Hargreaves BA, Vasanawala SS, Nayak KS, Hu BS, Nishimura DG. Fat-suppressed steady-state free precession imaging using phase detection. Magn Reson Med 2003;50:210-3. https://doi.org/10.1002/mrm.10488.

[36] Noll DC, Meyer CH, Pauly JM, Nishimura DG, Macovski A. A homogeneity correction method for magnetic resonance imaging with time-varying gradients. IEEE Trans Med Imaging 1991;10:629-37. https://doi.org/10.1109/42.108599.

[37] Irarrazabal P, Meyer CH, Nishimura DG, Macovski A. Inhomogeneity correction using an estimated linear field map. Magn Reson Med 1996;35:278-82. https://doi.org/10.1002/mrm.1910350221.

[38] Chen W, Meyer $\mathrm{CH}$. Fast automatic linear off-resonance correction method for spiral imaging. Magn Reson Med 2006;56:457-62. https://doi.org/10.1002/mrm.20973.

[39] Feng X, Salerno M, Kramer CM, Meyer CH. Non-Cartesian balanced steady-state free precession pulse sequences for real-time cardiac MRI. Magn Reson Med 2016;75:1546-55. https://doi.org/10.1002/mrm.25738. 


\section{CAPTIONS}

Table 1. Subjective image quality scores, from 1 to 5 , in all three categories. Median and interquartile range (IQR) are given for each category and each method. * indicates a statistically significant difference $(p<0.05)$.

Table 2. Ventricular volumes obtained from the BH-SAX and FB-WH acquisitions. Mean and standard deviation are given for each method, as well as bias and limits of agreement calculated using Bland-Altman analysis. Abbreviations: LV, left ventricle; RV; right ventricle; EDV, end diastolic volume; ESV, end systolic volume; SV, stroke volume; EF, ejection fraction. * indicates a statistically significant difference $(p<0.05)$.

Figure 1. Sampling strategies. Two sampling strategies were considered, both based in a nested loop strategy, with partitions in the inner loop and rotations in the outer loop. In US (uniform spacing), partition coordinates are in a Cartesian grid. In TS (tiny golden ratio spacing), partition spacing is based on the 7 th tiny golden ratio. In both strategies, spirals are rotated by the golden angle, $\theta$.

Figure 2. Respiratory navigator. Head-foot projections along time, obtained using the samples along the $k_{z}$ axis. Superimposed in white, the respiratory surrogate signal obtained via PCA and Fourier analysis.

Figure 3. Simulation study results. From top to bottom: fully sampled reference image, uniform step acquisition, tiny golden step acquisition. From left to right: axial, coronal views, and through time views of the numeric phantom.

Figure 4. Patient study results. Multiplanar reformation of a representative dataset in shortaxis orientation and corresponding breath-hold image. Both are depicted for end diastole and end systole.

Figure 5. Patient study results. Coronal view of a representative dataset in end-inspiration and end-expiration. The white line is aligned to the dome of the right hemidiaphragm in endinspiratory position for reference.

Figure 6. Bland-Altman plots for volumes of clinical interest: LV EDV, LV ESV, RV EDV and RV ESV. The continuous line indicates the bias and the dashed lines indicate the limits of agreement. 
Median \pm IQR / 2

\section{BH-SAX}

FB-WH

Sharpness of the endocardial border

$3.0 \pm 0.5 \quad 2.0 \pm 1.0^{*}$

Temporal fidelity of wall motion

$4.0 \pm 0.0 \quad 2.5 \pm 0.5^{*}$

Residual artefacts

$4.0 \pm 0.0$

$3.0 \pm 0.0^{*}$ 


\begin{tabular}{|c|c|c|c|}
\hline & \multicolumn{2}{|c|}{ Mean \pm standard deviation } & \multirow{2}{*}{ Bias (limits of agreement) } \\
\hline & BH-SAX & FB-WH & \\
\hline LVEDV (mL) & $150 \pm 53$ & $146 \pm 50$ & $-4.0(-23.6$ to 15.6$)$ \\
\hline LVESV (mL) & $54 \pm 23$ & $59 \pm 26^{*}$ & $+5.3(-4.4$ to 15.0$)$ \\
\hline LVSV (mL) & $96 \pm 32$ & $87 \pm 26^{*}$ & $-9.2(-27.2$ to 8.8$)$ \\
\hline LVEF (\%) & $65 \pm 6$ & $61 \pm 6^{*}$ & $-3.9(-9.7$ to 2.0$)$ \\
\hline RVEDV (mL) & $177 \pm 47$ & $169 \pm 48^{*}$ & $-8.2(-27.2$ to 10.8$)$ \\
\hline RVESV (mL) & $77 \pm 27$ & $79 \pm 25$ & $+2.6(-14.1$ to 19.2$)$ \\
\hline RVSV (mL) & $100 \pm 23$ & $89 \pm 24^{*}$ & $-10.9(-27.3$ to 5.5$)$ \\
\hline RVEF (\%) & $57 \pm 5$ & $54 \pm 3^{*}$ & $-4.0(-11.9$ to 4.0$)$ \\
\hline
\end{tabular}




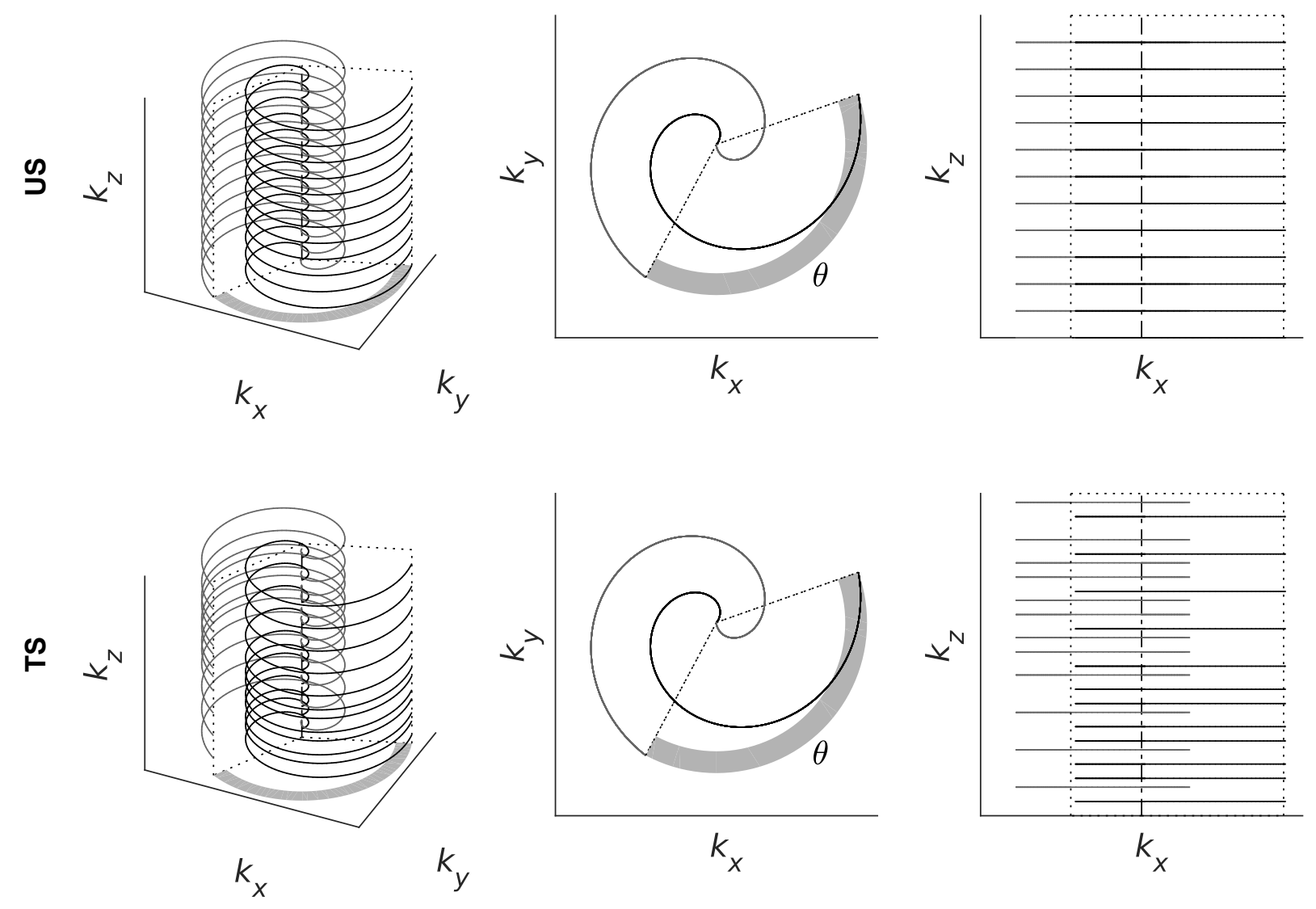




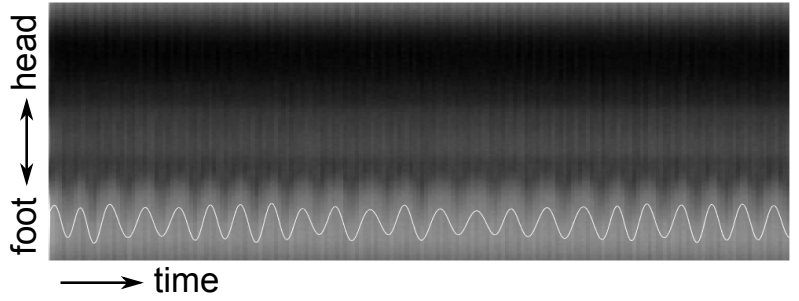




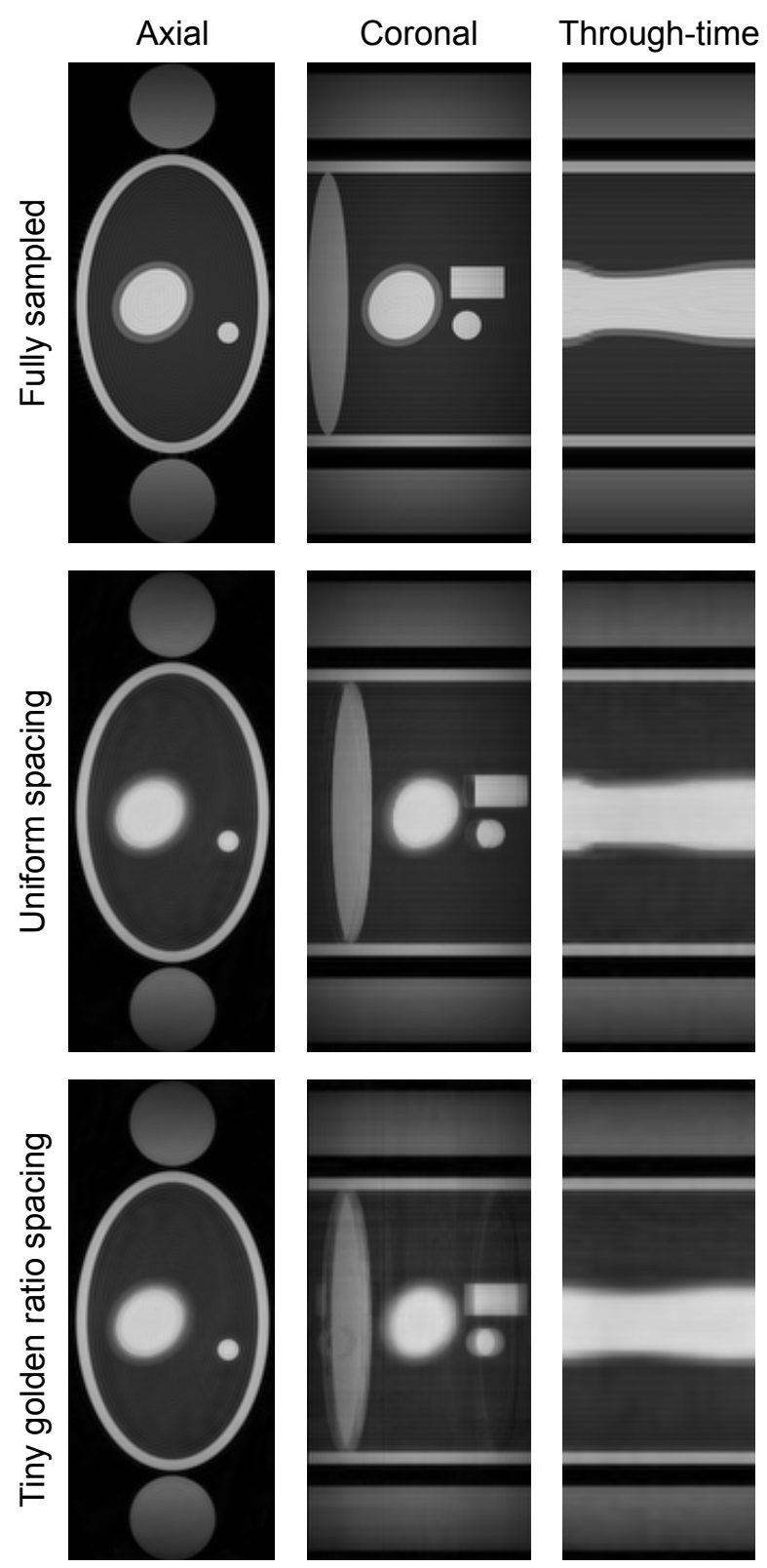


FB-WH

BH-SAX
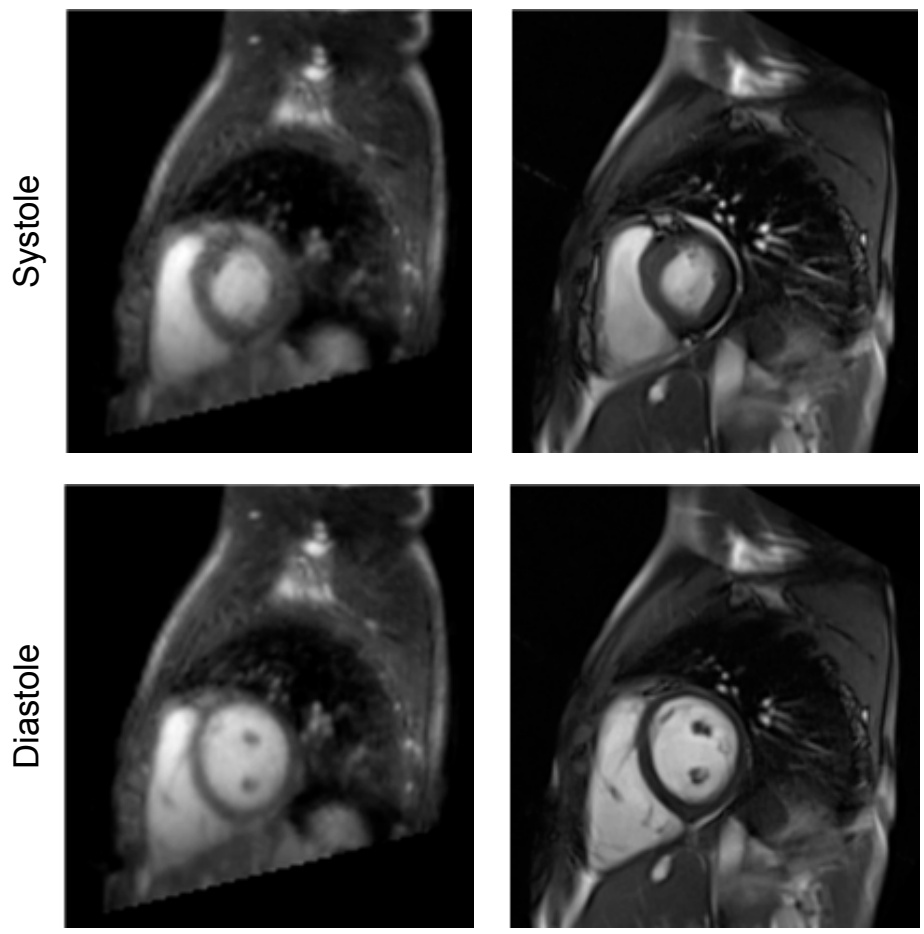


\section{End-inspiration}

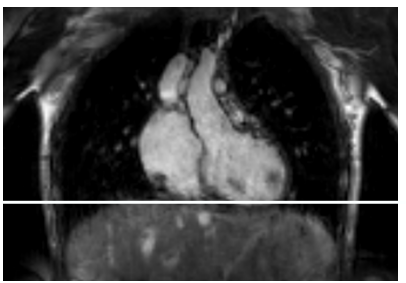

End-expiration

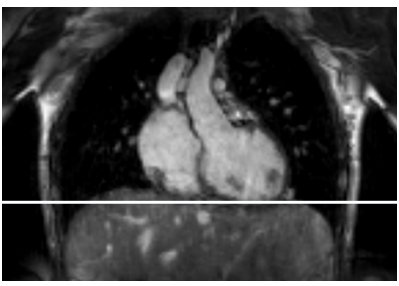




\section{LV}
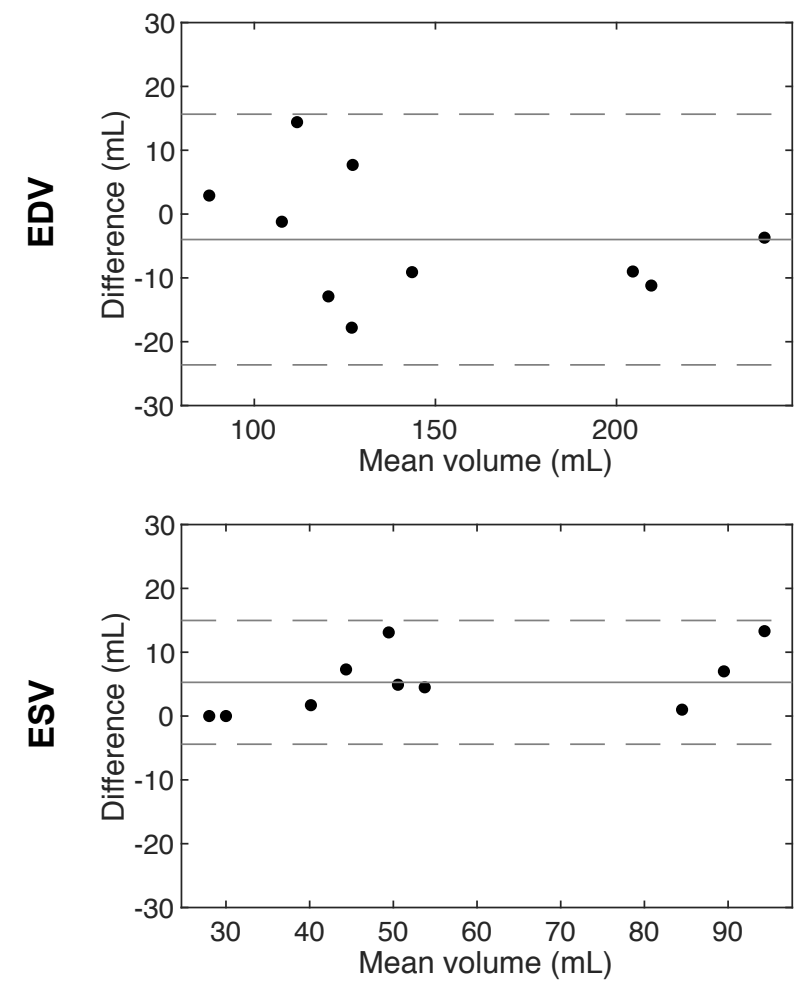

RV
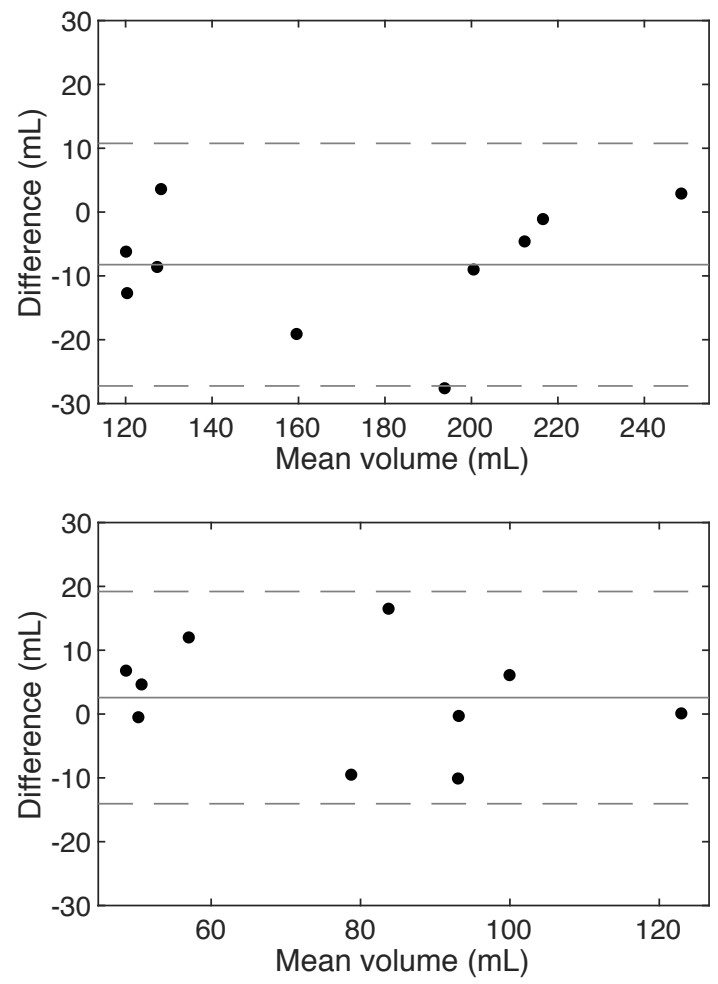


\section{Appendix A. Comparison with similar techniques}

The results in this work were compared to those of other relevant methods in the literature; in particular, other free-breathing isotropic 3D cine imaging techniques (see table below). Please note it is not the aim of this comparison to provide an extensive review of $3 \mathrm{D}$ cine imaging techniques, but rather to help put this work in context. The authors apologize for failing to include any other publications that might deserve inclusion.

Every effort has been made to present data fairly while facilitating comparison. However, caveats exist. Due to the complexity of techniques being compared, there are many uncontrolled sources of variability. Images have been analysed by different observers following different guidelines. Averages reported here are based on small samples. Subjective quality scores are defined differently in each case. Reconstructions were made in different computers at different optimization levels. Therefore, every care should be taken when drawing conclusions from these data. Nevertheless, it is hoped that the analysis will be useful to convey general ideas about the state of the art regarding free-breathing isotropic 3D cine cardiac imaging.

The work by Feng et al. [1] uses a radial koosh-ball trajectory (spiral phyllotaxis) and a cardiorespiratory resolved compressed sensing reconstruction, similar to the one used in this paper. It provides high quality images in terms of spatial resolution $(1.15 \mathrm{~mm})$, subjective scores and bias in volumetric measurements. However, this comes at a heavy cost in terms of scan time ( 14 min).

The other three techniques provide larger voxel size $(2.0 \mathrm{~mm})$ but also lower scan times ( 3$9 \mathrm{~min}$ ). Moghari et al. [2] employ a simple Cartesian trajectory and SENSE reconstruction combined with prospective motion compensation (re-acquiring data outside predefined acceptance window). This relatively straightforward acquisition takes longer than in other studies, but enables fast reconstruction without additional equipment or further optimization. They report relatively high subjective image quality and ventricular volume bias (see table below).

Usman et al. [3] also use a Cartesian trajectory but they incorporate sparsity constraints into their reconstruction (TV-SENSE). To suit this type of reconstruction, they use a reordering strategy (CASPR-Tiger) that aims for incoherent aliasing. They acquire a smaller number of cardiac phases (16 vs 20 for the other techniques considered here). Finally, they use softgating and rigid motion correction to improve scanning efficiency. For these reasons, scan 
time is rather low, which is traded off for increased reconstruction complexity. They report slightly lower subjective image quality and lower ventricular volume bias (see table below).

Finally, this work combines a stack of spirals trajectory, optimized for incoherent aliasing, with a cardiorespiratory resolved compressed sensing reconstruction similar to that used in Feng et al. This enables very fast acquisition: this work reports the lowest scan time. However, like in Feng et al., the reconstruction of high-dimensional images from non-Cartesian $k$-space data is very computationally expensive. Subjective image quality scores and ventricular measurements are similar to those in other studies. Images in this study score particularly low on sharpness (issues with blurriness are extensively discussed in main text) but do better on temporal fidelity and residual artefact. Ventricular volume biases (low for EDV, higher for ESV) are within the range of those reported elsewhere.

\section{References}

[1] Feng L, Coppo S, Piccini D, Yerly J, Lim RP, Masci PG, et al. 5D whole-heart sparse MRI. Magn Reson Med 2018;79:826-38. https://doi.org/10.1002/mrm.26745.

[2] Moghari MH, Barthur A, Amaral ME, Geva T, Powell AJ. Free-breathing whole-heart $3 \mathrm{D}$ cine magnetic resonance imaging with prospective respiratory motion compensation. Magn Reson Med 2018;80:181-9. https://doi.org/10.1002/mrm.27021.

[3] Usman M, Ruijsink B, Nazir MS, Cruz G, Prieto C. Free breathing whole-heart 3D CINE MRI with self-gated Cartesian trajectory. Magn Reson Imaging 2017;38:129-37. https://doi.org/10.1016/J.MRI.2016.12.021. 


\begin{tabular}{|c|c|c|c|c|c|c|c|c|c|c|}
\hline & \multirow{2}{*}{ Trajectory } & \multirow{2}{*}{ Reconstruction } & \multirow{2}{*}{$\begin{array}{l}\text { Respiratory } \\
\text { motion }\end{array}$} & \multirow{2}{*}{$\begin{array}{l}\text { Voxel } \\
\text { size } \\
(\mathrm{mm})\end{array}$} & \multirow{2}{*}{$\begin{array}{l}\text { Cardiac } \\
\text { phases }\end{array}$} & \multirow{2}{*}{$\begin{array}{l}\text { Subjective } \\
\text { image } \\
\text { quality } 1\end{array}$} & \multicolumn{2}{|c|}{$\begin{array}{l}\text { LV volume } \\
\text { bias (\%) }\end{array}$} & \multirow{2}{*}{$\begin{array}{l}\text { Scan } \\
\text { time } \\
\text { (min) }\end{array}$} & \multirow{2}{*}{$\begin{array}{l}\text { Reconstruction } \\
\text { time (min) }\end{array}$} \\
\hline & & & & & & & EDV & ESV & & \\
\hline $\begin{array}{l}\text { Feng et } \\
\text { al. } \\
(2018)\end{array}$ & $\begin{array}{c}\text { Radial } \\
\text { (spiral } \\
\text { phyllotaxis) }\end{array}$ & $\begin{array}{c}\mathrm{PICS}_{2} \text { (cardiac } \\
\mathrm{TV}+\text { respiratory } \\
\left.\mathrm{TV}_{3}\right)\end{array}$ & $\begin{array}{l}\text { Resolved (4 } \\
\text { phases) }\end{array}$ & 1.15 & 20 & $2.6-3.44$ & 1.1 & 3.3 & 14.3 & 408 \\
\hline $\begin{array}{l}\text { Moghari } \\
\text { et al. } \\
\text { (2018) }\end{array}$ & Cartesian & SENSE $_{5}$ & $\begin{array}{l}\text { Heart-NAV6 } \\
\text { (prospective) }\end{array}$ & 2.0 & $\begin{array}{c}20 \text { (30 by } \\
\text { interpolation) }\end{array}$ & $3.1,2.67$ & 9.5 & 4.0 & $\begin{array}{l}5.9 \pm \\
2.7\end{array}$ & Fast8 \\
\hline $\begin{array}{l}\text { Usman } \\
\text { et al. } \\
(2017)\end{array}$ & $\begin{array}{l}\text { Cartesian } \\
\text { (CASPR- } \\
\text { Tigerg) }\end{array}$ & $\begin{array}{l}\text { SENSE with } \\
\text { temporal TV }\end{array}$ & $\begin{array}{l}\text { Soft-gating + } \\
\text { motion } \\
\text { correction }\end{array}$ & 2.0 & 16 & $\sim 2.1, \sim 2.210$ & 2.7 & 3.7 & $4-5$ & 150 \\
\hline $\begin{array}{l}\text { This } \\
\text { work }\end{array}$ & $\begin{array}{c}\text { Spiral } \\
\text { (GRASS } 11)\end{array}$ & $\begin{array}{l}\text { PICS (cardiac TV } \\
\text { + respiratory TV) }\end{array}$ & $\begin{array}{l}\text { Resolved (4 } \\
\text { phases) }\end{array}$ & 2.0 & 20 & $\begin{array}{c}2.0,2.5 \\
3.012\end{array}$ & -2.7 & 9.3 & 3.6 & 106 \\
\hline
\end{tabular}

1 Exact definitions vary. Care should be taken when comparing these results directly.

2 Parallel imaging + compressed sensing reconstruction.

3 Total variation.

4 Image quality on a 0-4 Likert scale. Independent figures originally reported for different vessels, range is reported here.

5 Sensitivity encoding.

6 Image-based navigator with pre-defined acceptance window.

7 Sharpness of ventricular borders on a 1-4 Likert scale; LV and RV, respectively.

8 Fast in-line reconstruction on the scanner.

${ }_{9}$ Cartesian trajectory with spiral profile ordering and tiny golden step for eddy current reduction

10 Sharpness of endocardial border and residual artefact on 0-4 Likert scale. Approximate values taken from figure.

11 Golden ratio stack of spirals.

${ }_{12}$ Sharpness of endocardial border, temporal fidelity of wall motion and residual artefact on 0-4 scale. 